\title{
The Relaxation Effects Influence on the Formation of a Heat Flux in a Wall when a Viscous Shock Wave is Reflected from it
}

\author{
A. V. Panasenko \\ Ishlinsky Institute for Problems in Mechanics RAS, \\ Moscow, 119526, Russia \\ akpanas@mail.ru
}

\begin{abstract}
Results of calculation of heat flow in solid wall from iron or bismuth are given. Relaxation effects are taken into account on the example of viscous shock wave at Mach number of shock wave $\mathrm{M}=5$.
\end{abstract}

Keywords: relaxation effects, shock wave, heat flux.

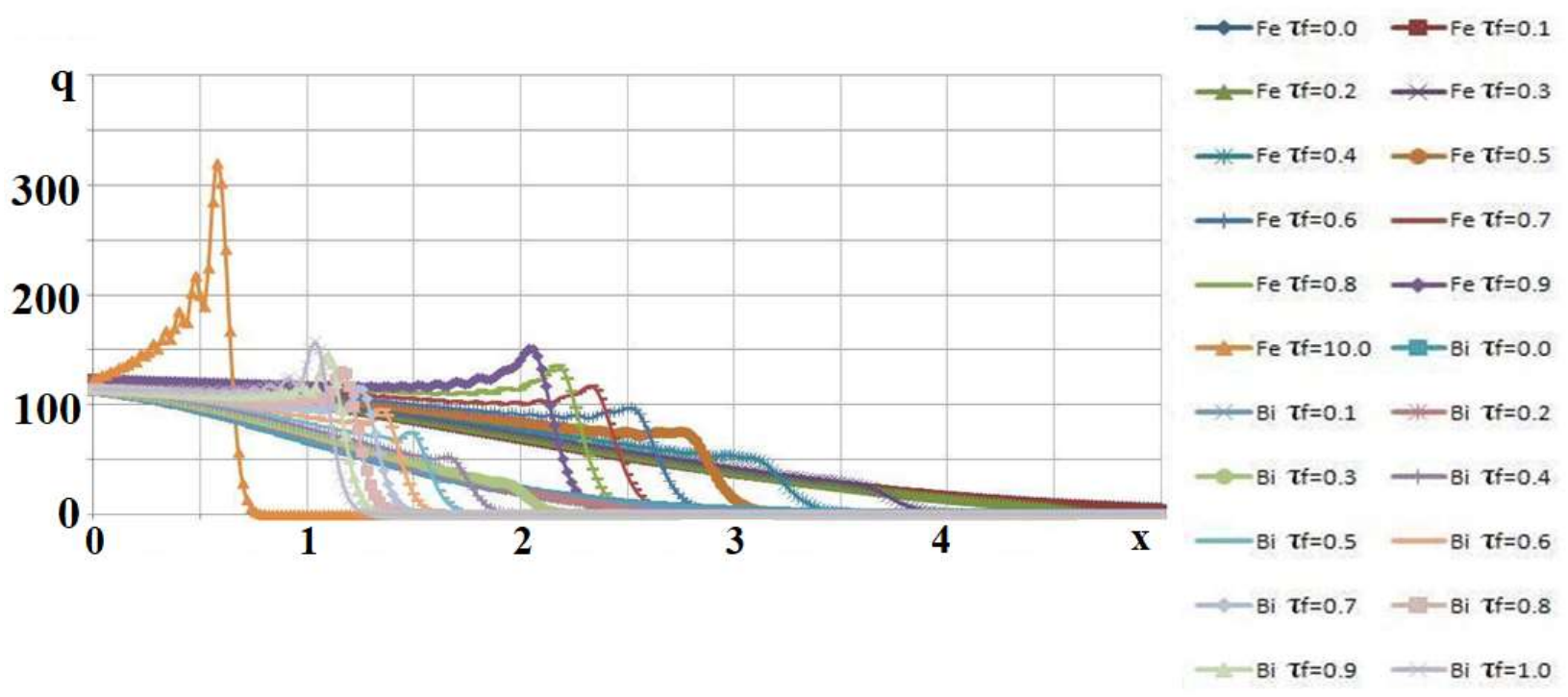

Profiles of heat flows from bismuth and iron at time $t=0.03371$

The results of calculating the heat flow in the wall of bismuth and iron when reflecting a viscous shock wave profile from it in the presence of relaxation properties showed that the heat front is formed the later the higher the relaxation coefficient. The noticeable lag of the thermal front in bismuth with respect to iron is associated with a significantly lower value of the thermal conductivity coefficient. 


\title{
Влияние релаксационных эффектов на формирование теплового потока в стенке при отражении от нее вязкой ударной волны
}

\author{
А. В. Панасенко \\ Институт проблем механики им. А.Ю. Иилинского Российской академии наук, \\ Россия, Москва, 119526, проспект Вернадского, д. 101-1 \\ akpanas@mail.ru
}

\begin{abstract}
Аннотация
Приведены результаты расчета теплового потока в твердой стенке из железа или висмута с учетом релаксационных эффектов на примере отражения от нее вязкой ударной волны при числе Маха ударной волны $\mathrm{M}=5$.

Ключевые слова: релаксационные эффекты, ударная волна, тепловой поток.
\end{abstract}

\section{1. Введение}

При рассмотрении процессов в средах считается общепринятым использование закона Фурье при записи уравнения энергии с учетом распространения тепла $[1,2]$. Важной отличительной особенностью среды с релаксационными свойствами является необходимость учета в ней запаздывания распространения тепла. Это обстоятельство приводит к необходимости использования в уравнении энергии при определении удельного теплового потока q вместо закона Фурье выражение вида

$$
q=k \frac{\partial T}{\partial x}-\tau \frac{\partial q}{\partial T},
$$

где $k$ - коэффициент теплопроводности; $T$ - температура; $x$ - продольная координата; $t-$ время; $\tau$ - эмпирическая константа (коэффициент релаксации), отражающая конечную скорость распространения теплового потока $[3,4]$.

При формировании профиля вязкой ударной волны при распаде разрыва газодинамических параметров отсутствует характерный линейный размер и характерное время процесса может быть вычислено следующим образом

$$
t_{g}=\frac{\eta}{P_{g}}=\frac{1.81 \times 10^{-5}}{101325} \sim 10^{-10} \mathrm{c}
$$

Характерное время $t_{g}$ находится в качественном соответствии с характерной величиной времени релаксационных процессов из $[3,4]$ и дает основание полагать, что при рассмотрении отражения вязкой ударной волны от стенки релаксационные эффекты могут оказывать заметное влияние на ход газодинамического процесса.

В работах [5] при решении уравнений Навье - Стокса было показано (рис. 1), что при отсутствии в среде эффектов релаксации $\tau=0$ (кривая 1) численное решение совпадает с аналитическим (точки) [6]. По мере усиления релаксационных процессов профиль ударной волны становится круче и в своей верхней части приобретает характерный изгиб, хорошо различимый на кривой 6 (кривые 2-6 соответствуют значениям $\tau: 2-0.2,3-0.4,4-0.6$, 5 - 0.8, 6-1.0). На рис. 1 кривые приведены совмещенными по средней точке профиля для 
более наглядной иллюстрации процесса формирования ударной волны при скорости ее движения с числом Маха $\mathrm{M}=5$.

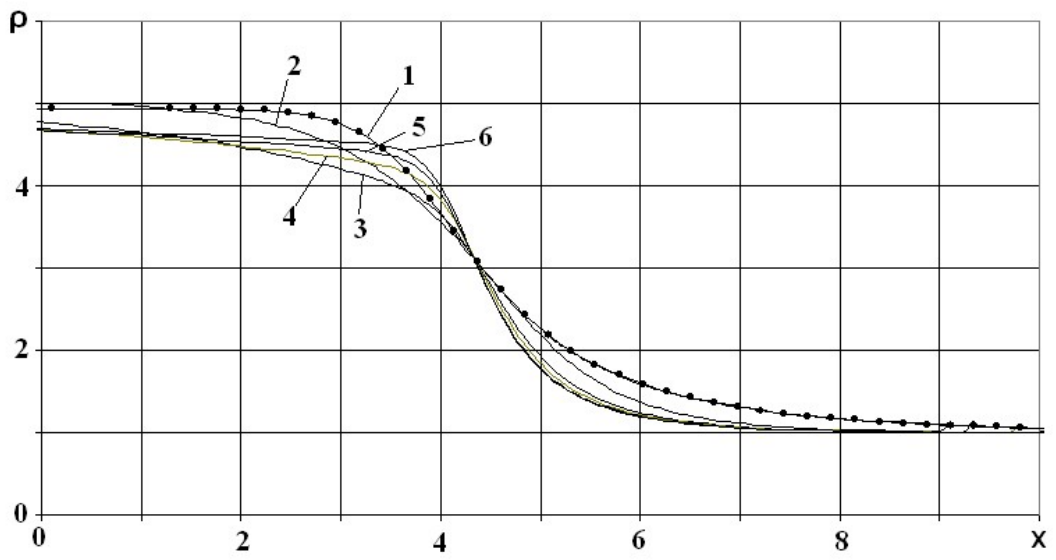

Рис. 1. Профили плотности в ударной волне в зависимости от величины коэффициента релаксации

Поэтому релаксационные эффекты могут влиять на профили теплового потока в твердой стенке в зависимости от свойств ее материала при отражении от нее вязкой ударной волны.

\section{2. Постановка задачи и методы расчета}

Рассмотрение задачи о формировании профиля ударной волны в вязком газе в строгой постановке [6] приводит к решению, зависящему от продольной координаты в диапазоне $-\infty<x<+\infty$. Поэтому при формулировке задачи о взаимодействии профиля ударной волны в вязком газе с теплопроводной стенкой имеет смысл ее рассмотреть в нижеследующей постановке.

Рассматриваемая задача является сопряженной, описываемая в газовой фазе системой уравнений Навье - Стокса [7]. а в твердом теле - уравнением распространения тепла [4]. Она определяется следующими характерными невозмущенными параметрами в газе: $\rho_{g}-$ плотностью; $P_{g}$ - давлением; $\eta$ - вязкостью; $k_{g}$ - коэффициентом теплопроводности; $C_{p g}-$ удельной теплоемкости давления; показателем адиабаты $\gamma=1.4 ; t_{g}-$ характерным временем процесса; $L$ - характерным линейным размером; числами Рейнольдса $-\operatorname{Re}=1$ и Прандтля $\operatorname{Pr}=0.75 ; \tau_{g}, \tau_{g g}-$ коэффициентом релаксации и безразмерным коэффициентом релаксации.

При численном решении задачи в качестве характерных масштабов были выбраны параметры в невозмущенной газовой фазе.

Определяющие задачу физические константы в стенке из металла: $\rho_{f}-$ плотность вещества стенки; $k_{f}$ - коэффициентами теплопроводности вещества стенки; $C_{f}-$ коэффициент удельной теплоемкости вещества стенки; $\tau_{f}, \tau_{f g}-$ коэффициент релаксации и безразмерный коэффициент релаксации стенки.

Величины $t_{g}, L, \tau_{g g}, \tau_{f g}$ имеют вид

$$
t_{g}=\frac{\eta}{P_{g}} ; \quad L=\frac{\eta}{\sqrt{\rho_{g} P_{g}}} ; \quad \tau_{g g}=\frac{\tau_{g}}{t_{g}} ; \quad \tau_{f g}=\frac{\tau_{f}}{t_{g}}
$$

Числовые значения определяющих задачу физических констант в газовой и твердой фазах, принятые при проведении расчетов.

Газовая фаза:

$\rho_{s}=1.293$ кг $/ \mathrm{M}^{3}, \eta=1.81 \times 10^{-5}$ кг/(м·с), $k_{g}=257 \times 10^{-4}$ вт/(м·град), $C_{p g}=1006$ дж/(кг·град); 
Железо: $\rho_{f}=7880$ кг/м³,$k_{f}=74.4$ вт/(м·град), $C_{f}=457$ дж/(кг·град);

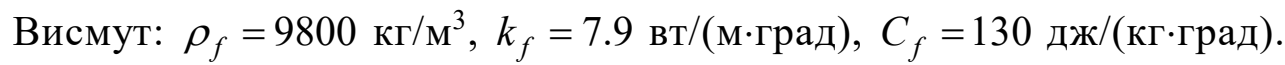

Влияние релаксационных эффектов среды на развитие нестационарного процесса рассмотрено на примере задачи (рис.2) о формировании теплового потока в области 3, возникающего в результате распада разрыва в газе (SS) в воздухе с параметрами, соответствующими, формированию профиля ударной волны в областях 1 и 2 и последующего его отражения от теплопроводной стенки (WW) из железа или висмута.

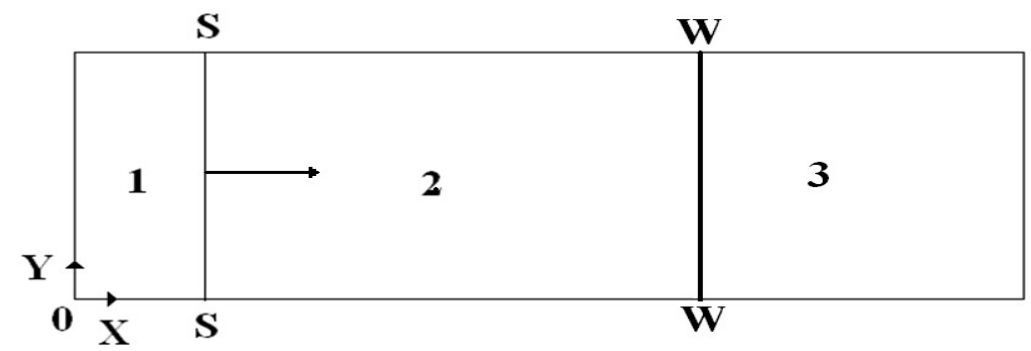

Рис. 2. Схема постановки задачи

При проведении расчетов в традиционных обозначениях в областях 1, 2 (рис. 2) использована система уравнений Навье-Стокса, описывающая нестационарные течения вязкого теплопроводного газа, записанная в декартовой прямоугольной системе координат в интегральной форме [7]. Учет вязких членов рассчитывался с помощью разложения в ряд Тейлора на соседних с границей расчетных ячейках. Учет релаксационных членов в уравнении энергии поясним на примере.

$$
\begin{gathered}
\frac{\partial E}{\partial t}+\frac{\partial G}{\partial x}=-\frac{1}{(\gamma-1) M^{2} \operatorname{Re} \times \operatorname{Pr}} \times \frac{\partial q}{\partial x}, \\
\frac{\partial q}{\partial t}=-\frac{1}{\tau_{g g}}\left(\frac{\partial T}{\partial x}+q\right)
\end{gathered}
$$

Значение $q^{n}$ в уравнении энергии находится по значению $q^{n+1}$ из второго уравнения на предыдущем временном слое.

При проведении расчетов все газодинамические параметры отнесены к невозмущенным значениям: давления $P_{0}$, плотности $\rho_{0}$, температуре к $T_{0}=300 \mathrm{~K}$, координаты - к характерной длине $L$.

Расчеты нестационарного течения в областях 1, 2 (рис. 2) проведены с использованием модифицированной разностной схемы типа МакКормака [8]. Модификация разностной схемы заключалась в последовательном применении на шаге интегрирования ее шаблона в прямом и зеркальном отображении с соответствующей переписью ее формул. Такая модификация позволяет построить более симметричный по результатам интегрирования шаблон разностной схемы. Схема МакКормака имеет второй порядок точности, что предполагает введение в алгоритм расчета монотонизирующего решение оператора сглаживания, предложенного в [9].

$$
\begin{gathered}
\vec{f}_{i}^{n+1}=\vec{f}_{i}+\frac{\vec{\varphi}_{i+1 / 2}^{*}-\vec{\varphi}_{i-1 / 2}^{*}}{\Delta \Omega_{i}}, \\
\mid \vec{\varphi}_{i+1 / 2}^{*}= \begin{cases}\vec{\varphi}_{i+1 / 2}, & \text { если }\left(\delta \rho_{i+1 / 2} \cdot \delta \rho_{i-1 / 2}<0\right) \cup\left(\delta \rho_{i+1 / 2} \cdot \delta \rho_{i+3 / 2}<0\right), \\
0, & \text { в противном случае }\end{cases}
\end{gathered}
$$




$$
\begin{gathered}
\vec{\varphi}_{i+1 / 2}=Q \times \delta \vec{f}_{i+1 / 2}^{n} \times \psi\left(\Delta \Omega_{i} \times \Delta \Omega_{i+1}\right), \\
\delta \vec{f}_{i+1 / 2}=\vec{f}_{i+1}-\vec{f}_{i}, \quad \psi(a, b)=\frac{2}{\frac{1}{\Delta \Omega_{i}}+\frac{1}{\Delta \Omega_{i+1}}}
\end{gathered}
$$

Здесь $f$ - полученная после монотонизации параметров в ячейке функция; $\varphi$ - полученная в расчете газодинамическая функция; $\Omega$ - объем расчетной ячейки; $\rho$ - плотность газа в ячейке; $Q$ - эмпирически подобранный коэффициент ( $Q=0.2)$.

Смысл использования оператора сглаживания поясняет рис. 3. При возникновении колебания решения по плотности газа оператор сглаживания приводит к уменьшению пика колебания, при отсутствии колебания плотности - монотонизация решения не проводится. При наличии колебания решения к функции добавляется вторая производная, что приводит к уменьшению колебания, что очевидно для однородной сетки.

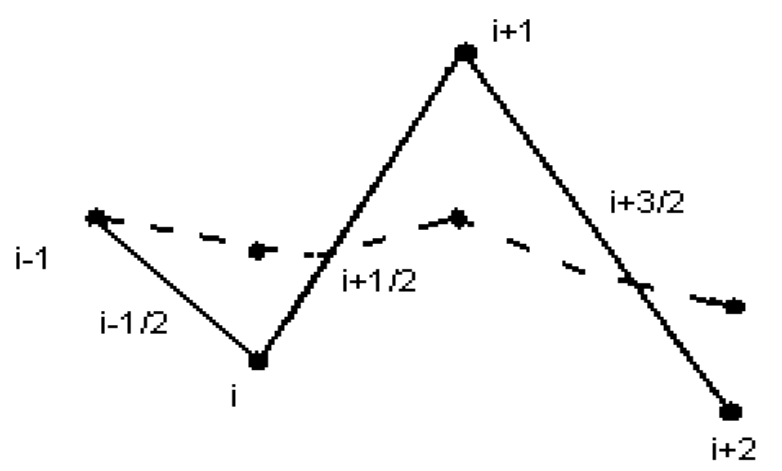

Рис. 3. Результат работы оператора сглаживания решения

Уравнения, описывающие процесс распространения тепла в твердом теле имеют вид

$$
\begin{gathered}
\frac{\partial T}{\partial t}=-\frac{1}{\operatorname{Re} \times \operatorname{Pr}} \times \frac{\rho_{g} C_{p g}}{\rho_{f} C_{f}} \frac{\partial q}{\partial x}, \\
\tau_{f g} \frac{\partial q}{\partial t}=-\frac{k_{f}}{k_{g}} \frac{\partial T}{\partial x}-q
\end{gathered}
$$

Численный метод решения уравнений теплопроводности в стенке в единых масштабах имеет вид

$$
\begin{gathered}
T_{i}^{n+1}=T_{i}^{n}-\frac{1}{\operatorname{Re} \times \operatorname{Pr}} \times \frac{\rho_{g} C_{p g}}{\rho_{f} C_{f}} \times \frac{\Delta t}{\Delta x_{f}}\left(q_{i+1}^{n+1}-q_{i}^{n+1}\right), \\
q_{i}^{n+1}=\frac{\tau_{f g} q_{i}^{n}-\frac{\Delta t}{\Delta x_{f}} \frac{k_{f}}{k_{g}}\left(T_{i+1}^{n}-T_{i}^{n}\right)}{\Delta t+\tau_{f g}}
\end{gathered}
$$

В качестве начальных условий задавались:

- $\quad$ сформированный профиль ударной волны, как на рис. 1;

- $\quad$ параметры начальной температуры во всех расчетных ячейках в области 3.

В качестве граничных условий задавались:

- $\quad$ при $x=0$ параметры за фронтом ударной волны при заданном числе еe Maха;

- $\quad$ на твердой стенке WW - равенство тепловых потоков 


$$
-k_{g} \frac{\partial T_{g}}{\partial x_{g}}-\tau_{g g} \frac{\partial q_{g}}{\partial t}=-k_{f} \frac{\partial T_{f}}{\partial x_{f}} \tau_{f g} \frac{\partial q_{f}}{\partial t}
$$

- $\quad$ на правой границе расчетной сетки - по потоку $\mathrm{d} f / \mathrm{d} x=0$.

\section{3. Результаты расчетов}

На рис. 4 и 5 приведено сравнение мгновенных профилей тепловых потоков в стенке из железа при $\mathrm{M}=5$ при различных значениях коэффициента релаксации в различные моменты времени.

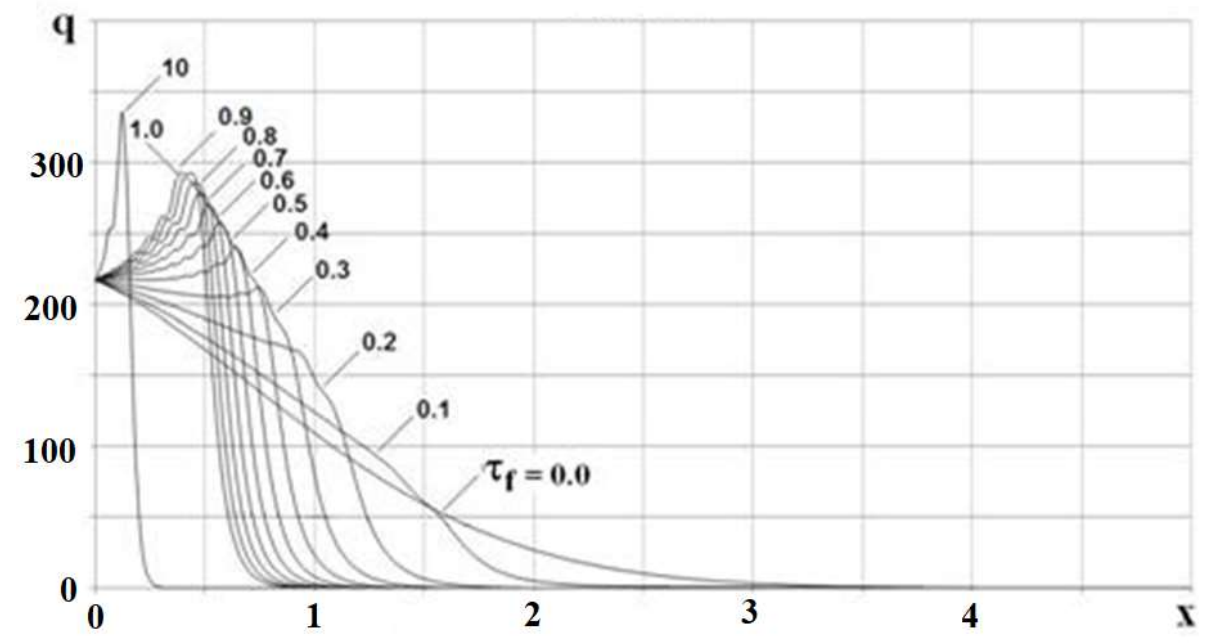

Рис. 4. Профили тепловых потоков из железа в момент времени $t=0.02072$

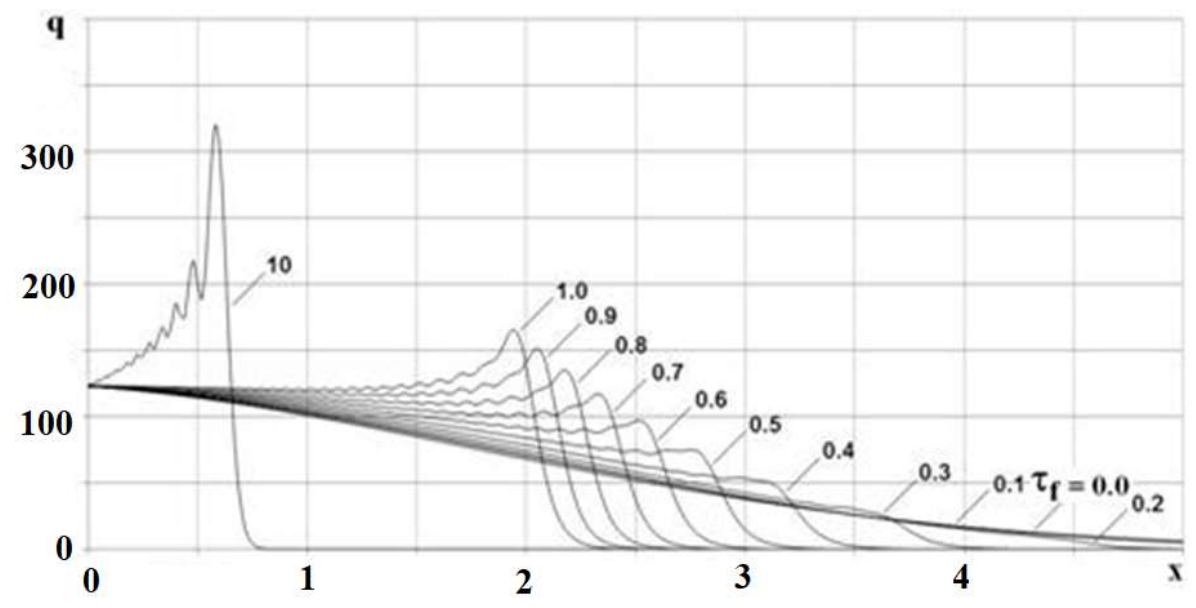

Рис. 5. Профили тепловых потоков из железа в момент времени $t=0.03371$

Видно, что наличие релаксационных свойств среды приводит к формированию в ней ярко выраженного теплового фронта тем позднее, чем выше коэффициент релаксации. По мере уменьшения амплитуды теплового фронта влияние релаксационных свойств среды ослабевает и мгновенный профиль теплового потока приближается к профилю теплового потока при отсутствии релаксационных эффектов (при $\tau_{f}=0$ ).

Сравнение мгновенных профилей тепловых потоков в стенках из железа и висмута при $\mathrm{M}=5$ при различных значениях коэффициента релаксации $\tau_{f}$ (рис. 6).

Видно, что в металлах с различными теплофизическими свойствами наличие релаксационных свойств среды приводит к качественно схожему формированию в ней ярко выраженного теплового фронта. 


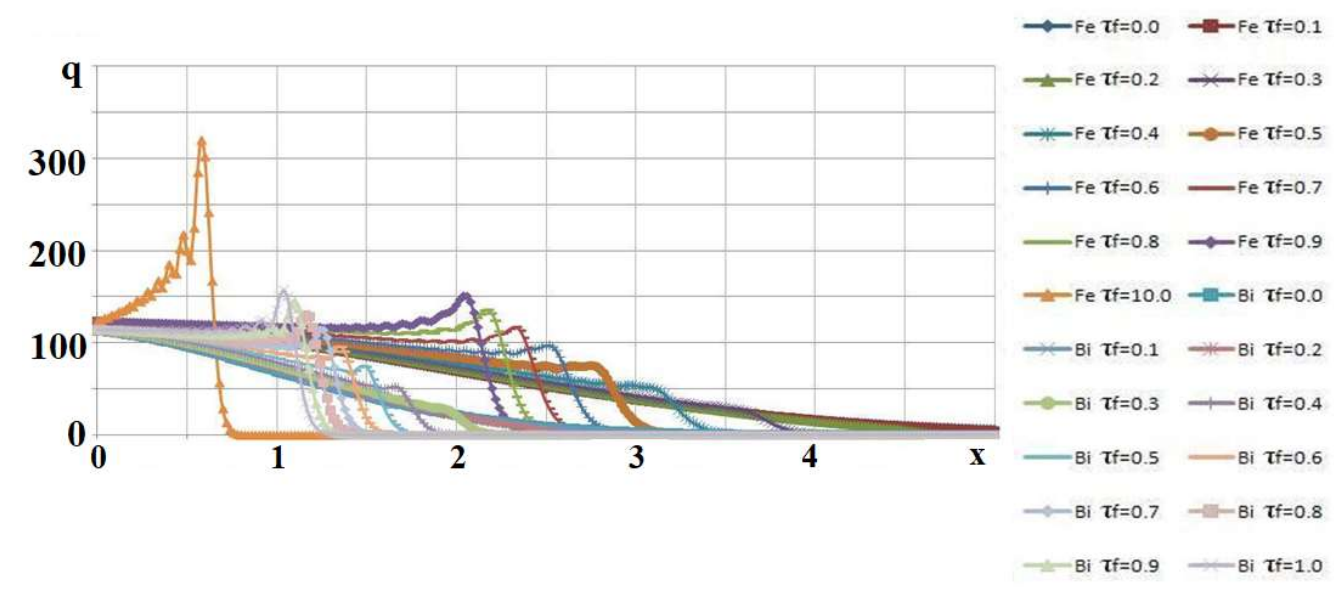

Рис. 6. Профили тепловых потоков из висмута и железа в момент времени $t=0.03371$

\section{4. Заключение}

Результаты расчета теплового потока в стенке из висмута и железа при отражении от нее профиля вязкой ударной волны при наличии релаксационных свойств показали, что тепловой фронт формируется тем позднее, чем выше коэффициент релаксации. Заметное отставание теплового фронта в висмуте по отношению к железу связано с существенно меньшим значением коэффициента теплопроводности.

Работа выполнена с выполнением расчетов на суперкомпьютере МСЦ РАН.

\section{Литература}

1. Котов М.А., Рулева Л.Б., Солодовников С.И., Суржиков С.Т. Исследование ударно-волновых процессов обтекания клиновидных моделей с кавернами в гиперзвуковой ударной аэродинамической трубе//Физико-химическая кинетика в газовой динамике. 2014. Т. 15, вып. 3. http://chemphys.edu.ru/issues/2014-15-3/articles/223/

2. Суржиков С.Т. Численное моделирование ударно-волнового взаимодействия с ламинарным пограничным слоем при гиперзвуковом обтекании моделей с изломом образующей/Физикохимическая кинетика в газовой динамике. 2019. Т. 20, вып. 4.

http://chemphys.edu.ru/issues/2019-20-4/articles/892/

3. Лыков А.В. Тепломассообмен. Справочник. М.: Энергия. 1978.

4. Котляр Я.М., Совершенный В.Д., Стриженов Д.С. Методы и задачи тепломассообмена. М.: Машиностроение. 1987.

5. Липницкий Ю.М., Панасенко А.В. Формирование профиля ударной волны в газе с учетом релаксационных эффектов // Письма в ЖТФ, 2009, том. 35, вып. 21, С. 57 - 60.

6. Becker R. // Zeitchr. fur Phys. 8 (1921-1922), pp. 321-322.

7. Андерсон Д., Таннехилл Дж., Плетчер Р. Вычислительная гидромеханика и теплообмен: Издво «Мир». Т. 1, 2. 1990.

8. Mac-Cormak R.W. The effect of viscosity in hypervelocity impact cratering // AIAA Paper. 1969. Pp. 69-354.

9. Жмакин А.И., Фурсенко А.А. Об одной монотонной разностной схеме сквозного счета // Ж. вычисл. матем. и матем. физ. 1980. Т. 20. № 4. С. 1021-1031.

\section{Reference}

1. Kotov, M. A., Ruleva, L. B., Solodovnikov, S. I., Surzhikov, S. T., "Investigation of shock wave process of model flow in hypersonic aerodynamic shock tube," Physical-Chemical Kinetics in Gas Dynamics, Vol. 15, No. 3, 2014. http://chemphys.edu.ru/issues/2014-15-3/articles/223/ 
2. Surzhikov, S. T., "Numerical modeling of shock-wave interaction with a laminar boundary layer during hypersonic flow around models with a kink," Physical-Chemical Kinetics in Gas Dynamics, Vol. 20, No. 4, 2019. http://chemphys.edu.ru/issues/2019-20-4/articles/892/

3. Lykov, A. V. Teplomassoobmen (Heat and mass transfer), Spravochnik, M.: Jenergija, 1978.

4. Kotljar, Ja. M., Sovershennyj, V. D., Strizhenov, D. S. Metody i zadachi teplomassoobmena (Methods and tasks of heat and mass transfer), M.: Mashinostroenie, 1987.

5. Lipnickij, Ju. M., Panasenko, A. V., "Formirovanie profilja udarnoj volny $\mathrm{v}$ gaze $\mathrm{s}$ uchetom relaksacionnyh jeffektov," Pis'ma v ZhTF, Vol. 35, No. 21, 2009, pp. 57-60.

6. Becker, R., "Stoßwelle und Detonation," Zeitschrift für Physik, Vol. 8, No. 1, 1922, pp. 321-362.

7. Anderson, D., Tannehill, Dzh., Pletcher, R. Vychislitel'naja gidromehanika i teploobmen (Computational fluid mechanics and heat exchange), Izd-vo «Mir», Vol. 1, 2, 1990.

8. Mac-Cormak, R.W., "The effect of viscosity in hypervelocity impact cratering," 4th Aerodynamic Testing Conference, AIAA Paper, April 1969, pp. 69-354.

9. Zhmakin, A. I., Fursenko, A. A., "Ob odnoj monotonnoj raznostnoj sheme skvoznogo scheta," $Z h$. Vychisl. Matem. i Matem. Fiz., Vol. 29, No. 4, 1980, pp. 1021-1031.

Статья поступила в редакцию 21 декабря 2020 г. 